\title{
Transient Brainstem Anaesthesia Following a Diagnostic Block for Trigeminal Neuralgia
}

\section{TO THE EDITOR}

Glycerol trigeminal rhizotomy is an established alternative ablative technique to radiofrequency thermocoagulation for patients with medically refractory trigeminal neuralgia (TN) (1). Complications following such percutaneous procedures, though described, are rare. We describe an occurrence of transient brainstem anaesthesia following diagnostic block for TN.

A 45-year-old man presented with a 3 year history of pain on the right side of his face. He was treated with Carbamazepine 800 mg/d, Pregabalin 150 mg/d, and Amitriptyline $25 \mathrm{mg} / \mathrm{d}$. As the pain persisted with drug side-effects, alternative treatment, including surgery, was offered. He opted for glycerol rhizotomy. Visual-analog-score (VAS) prior to the procedure was $90 \mathrm{~mm}$ with right $\mathrm{V} 3$ dermatomal distribution of severe intensity, pin-prick in nature, and frequent exacerbations. During the procedure, monitoring included electrocardiogram, pulse-oximetry, and non-invasive blood pressure. Using bi-plane fluoroscopic guidance, cannulation of right foramen ovale was attempted. Fluoroscopy confirmed position of the needle (Fig. 1). As there was no cerebro-spinal fluid (CSF) egress from the needle, repositioning was attempted. Despite 4 attempts, CSF flow was absent and suboptimal needle placement was suspected. A diagnostic block with $0.8 \mathrm{~mL}$ of lignocaine $2 \%$ was performed, which resulted in bradycardia (58/minute). The procedure was abandoned and the patient was shifted to an observation area. Post-procedure, the patient had hypoesthesia in ipsilateral V3/N2 region but complained of giddiness and nausea. Blood pressure was $174 / 104 \mathrm{mmHg}$ and heart rate was 64/minute. He was administered ondensetron $8 \mathrm{mg}$ intravenously and maintained in supine position with continuous monitoring. Simultaneously, the patient developed profuse sweating, multiple episodes of vomiting, and horizontal nystagmus. Suspecting intracranial bleed, a computed tomography (CT) scan was performed, which was normal. The patient was admitted for further observation and closely monitored for hemodynamic and neurological parameters. Over the next 4 hours, hemodynamic parameters stabilized, nystagmus and vomiting stopped. There was no giddiness

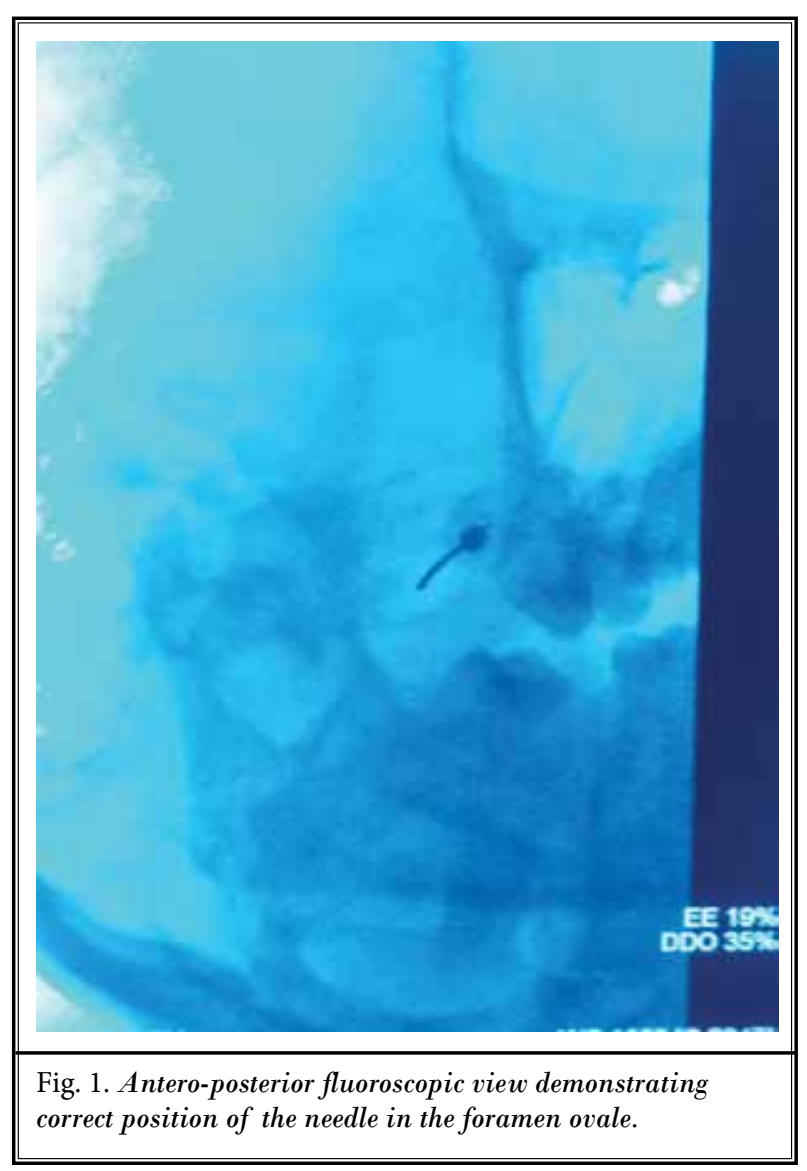

on ambulation and the patient was discharged home with instruction to visit the emergency room if new symptoms developed. At one week follow-up visit, VAS score had reduced to $30 \mathrm{~mm}$ without post-procedural adverse sequel.

Though CSF egress is a definitive sign of needle entry into trigeminal cistern, it is not always required for successful treatment for TN. However, its presence provides reassurance along with fluoroscopic confirmation during glycerol rhizotomy. In an attempt to obtain CSF egress, multiple attempts were made. As this was unsuccessful, a diagnostic local anaesthetic block was performed, which resulted in this complication. There are only a few reports of complications like vertigo and 
ataxia following alcohol neurolysis of mandibular nerve (2), trigemino-cardiac reflex (3), and brainstem anaesthesia with respiratory arrest following percutaneous procedures for TN (4). As the CT scan was normal and manifestations reversed within few hours, transient reversible local anaesthetic effect on the brainstem was the mostly likely cause for this complication in our patient. The probable mechanism is intrathecal spread of anaesthetic despite absence of CSF egress during the procedure. We discontinued the procedure after bradycardia occurred. Had the glycerol injection been performed, any adverse effect could have been long lasting.

To conclude, brainstem anaesthesia can occur after a diagnostic block for TN. Awareness about the same and vigilant monitoring will minimize adverse consequences.

Kamath Sriganesh, DM

Associate Professor

Department of Neuroanaesthesia

NIMHANS

Bangalore, India 560029

Email: drsri23@rediffmail.com
Manish Ranjan, MCh

Department of Neurosurgery

NIMHANS

Bangalore, India 560029

E-mail: drmanishranjan@gmail.com

Sudhir Venkataramaiah, MD

Department of Neuroanaesthesia

NIMHANS

Bangalore, India 560029

E-mail: vsudhir77@gmail.com

Sampath Somanna, MCh

Department of Neurosurgery

NIMHANS

Bangalore, India 560029

E-mail: sampath@nimhans.kar.nic.in

\section{References}

1. Udupi BP, Chouhan RS, Dash HH, Bithal 2 . PK, Prabhakar H. Comparative evaluation of percutaneous retrogasserian glycerol rhizolysis and radiofrequency thermocoagulation techniques in the management of trigeminal neuralgia. 3 Neurosurgery 2012; 70:407-412.
Chaturvedi A, Dash HH. Prolonged vertigo and ataxia after mandibular nerve block for treatment of trigeminal neuralgia. J Anaesth Clin Pharmacol 2011; 27:386-388.

3. Chowdhury T, Cappellani RB, Schaller B. Retrogasserian Glycerol Rhizolysis: First description of occurrence trigeminocardiac reflex. J Neurosurg Anesthesiol 2014; 26:86-87.

4. Nique TA, Bennett CR. Inadvertent brainstem anesthesia following extraoral trigeminal $V_{2}-V_{3}$ blocks. Oral Surg Oral Med Oral Pathol 1981; 51:468-470. 